\title{
Behavioural Approach for the Simulation of Electrical Consumption at the Neighbourhood Level
}

\author{
Eric Ferreri, Jean-Marc Salotti, Pierre-Alexandre Favier \\ IMS Laboratory, CNRS, Bordeaux INP, Bordeaux University \\ Bordeaux, France \\ eric.ferreri@ensc.fr
}

\begin{abstract}
We address the problem of simulating domestic activities related to electrical consumption. Continuously growing electrical energy requirements have become an issue as resources to produce it are limited. Human factors have to be taken into account in order to better predict electric consumption and optimize its production. We propose a framework based on a psychological scheme and on stochastic features. The decision process is based on the representation of habits and the use of heat maps. Inclusion of planning-related behaviour is performed by a non-parametric voting method using relational properties among domestic activities. The results show a good fitting between the predictions and ground truth.
\end{abstract}

Keywords - Simulation; bio-inspired model; heat map; hotspot; attractor

\section{INTRODUCTION}

We address the problem of simulating domestic electric consumption.

Newly-built habitations must conform to recent laws and regulations that impose efficiency levels related to energy consumption. For instance, new French norms such as RT 2012 require for each new habitation that energy consumption predictions for heating, illuminating, air conditioning and hot water must be below $50 \mathrm{kWh} / \mathrm{m}^{2}$ per year. In order to make sure that requirements are met, simulations are run to provide estimates of energy efficiency. The issue is that actual energy consumptions are often well above predicted figures. Inhabitant behaviour is usually singled out as a factor that is particularly difficult to predict and responsible for inaccuracies in estimations of energy consumptions. Usage of heating devices mainly depends on physical parameters such as outside temperatures and insulating characteristics [1]. In the present study, it is proposed to focus on electrical consumption in the context of household appliances. Their relative part in the total electric consumption is continuously increasing and hardly predictable due to the impact of human factors.

In recent years, energy savings measures have made the end-user increasingly important. He also has an influence on the whole distribution grid. For example, for distributed electricity generation in low-voltage grids, widespread onsite generation has important effects on local electricity networks and it is very difficult to match this with domestic electricity demand [2].

Modelling the domestic electricity demand for individual households is a complex task. The load profile of a single building strongly fluctuates. This high variability implies that it is very hard for power plants to efficiently meet the requirements of final users. Aggregating the demand of a number of buildings tends to create an over-smoothed profile that is much more predictable than its component parts (e.g., small neighbourhoods or individual residences). Taking account of this phenomenon is relevant to improve the quality of matching the energy demand and supply from onsite production. Appropriate predictions allow some reductions in local storage needs (or energy sold to the grid) and some reductions in the global energy cost. In order to generate accurate predictions, it is important to develop behavioural models. The identification of habits and usages helps to predict peaks of consumption. Most state-of-the-art methods combine information based on consumption statistics and simple models of human behaviour [3].

However, important differences exist among methods concerning the features and framework. Feature selection is a key issue to detect habits. A balanced trade-off must be found between performance and computational resources (time and storage space). Markov-chain methods have been proposed [4]. More discriminant methods, for instance based on inverse functions, can give very precise results [3].

An important issue is that technologies are evolving, habits are changing, new norms are appearing, and motivations and beliefs concerning ecology and sustainable development are increasingly taken into account in the human decision process. Hence the statistical approach based on past data is biased and care must be taken in the detection of trends. Decision-making models state that decisions are the result of an interaction between a cognitive structure (i.e., brain circuits) and surrounding data. The cognitive structure, which is largely independent from past data, provides more cues for the understanding of consumption data. The integration of behavioural data into a psychological scheme is a possible approach to approximate cognitive processes that ultimately lead to decisions and actions. The objective is to achieve better predictions of electrical consumption without the need for exhaustive databases and without loss of generality. 
We propose combining habit-related constraints with a psychological decision-making model. Two types of behaviours can be taken into account: Habits, which are usually performed at the same time of the day, and planned activities, which are linked to other activities.

We then present the psychological model and its computational implementation. In the third section, the use of heat maps is introduced to represent decisions concerning domestic activity. It is shown that habits and plans can be identified as specific features of the heat maps. The results are presented and discussed in the fourth section and the remaining issues are given in the conclusion.

\section{Methodology}

\section{A. Main issues}

In addition to simulations based on mathematical tools or statistical analysis of data, a multi-agent system allows an intuitive way to simulate behaviours. An agent is an autonomous structure that aims to mimic human actions.

Reactive agents are able to give stereotyped answers to an external stimulus. There is no symbolic representation and no reasoning (i.e., the agent turns the light on at $11 \mathrm{pm}$ ). More complex agents aim at using generic cognitive schemes to link electric consumption with behavioural variables. Belief Desire Intention agents (BDI) have been used by Kashif et al. to simulate actions at home. In their framework, beliefs refer to what is known about the surroundings (i.e., a room is too dark), desires are predefined as a set of needs that have to be fulfilled (i.e., minimal room illumination) and intentions are the set of actions that may be chosen to fulfil a particular desire (i.e. to turn the light on).

BDI agents are able to implement basic reasoning, with custom behavioural rules in a multi-agent system. Interactions and communications between agents can also be simulated. However, the generic BDI structure is not optimal when there is a need to simulate habits and motivated decisions. As habits are behaviours repeated in consistent settings, they provide an adequate answer to a well-known situation, without requiring attentional resources [5], whereas motivated decisions help to face specific situations that require some planning. The classical structure of BDI agents is not adapted to simulate several levels of thought processes. Thus we propose to use a scheme directly issued from psychological research at the core of the agent.

\section{B. Presentation of the psychological scheme}

Without reinforcement, there can be no habits. When considering domestic habits, the outcome of the performed action is seen as a reward that reinforces the habit (e.g., the reward for using the vacuum cleaner is having a clean carpet). An interesting approach to define and quantify the stability of a given habit has been proposed by Wood et al. [6].

An important source of variability in the decision space is linked to interactions among habits. We will illustrate this with an example. It is useful to iron clothes only once they are clean, so a delay in washing clothes will produce a delay in ironing them. In this case, the action of washing clothes "attracts" the action of ironing clothes at a later time of realization. We can say that washing clothes is an attractor to ironing, but the inverse is not true: the time of ironing has no effect on the time of washing, thus activities only interact in a certain order. We assume that this ordered interaction is what is usually called a plan: a succession of activities that are intentionally directed toward a desired outcome, whereas habits can be defined as actions that are initiated mostly by the context.

So far two cognitive strategies have been presented, reactive and goal-directed. Furthermore, the inclusion of intention in the definition of planning suggests the use of a psychological model to achieve appropriate and realistic modelling. The continuum model proposed by Fiske et al. is particularly interesting because it takes into account diverse levels of cognitive processes that lead to reactive habits and planned actions $[7,8]$. These cognitive processes are usually seen as qualitatively different but the model defines a continuum between them.

The original model is presented Figure 1. Fiske explains it as follows [8]: (1) perceivers initially categorize the environment and their immediate responses may be triggered by this first categorization; (2) if the target is at least minimally relevant, perceivers' further attention to attribute information will be determined by informational and motivational factors; (3) attention to attributes in turn mediates use of the processes along the continuum that follows; (4) when possible, perceivers will confirm the initial category; (5) when an initial category cannot be confirmed, perceivers will recategorize according to a subcategory, new category, exemplar, or self-category; (6) when recategorization fails, perceivers will use fully individuated, attribute-by-attribute, piecemeal integration processes. Finally, perceivers may decide that further assessment is needed and the global cognitive process repeats.

A computational model of the psychological scheme has been built to simulate electrical consumption, see Figure 2 .

The environment refers to the variables that have a direct impact on the use of appliances (e.g., time, amount of dirty clothes, amount of dirty plates, etc.).

The different levels of categorization refer to the different cognitive processes that take environment variables and past activities into account in order to make the next decision.

Habits and planned activities should be implemented in the current framework. We propose to analyse times series related to consumption data to explain the different categorization processes. 


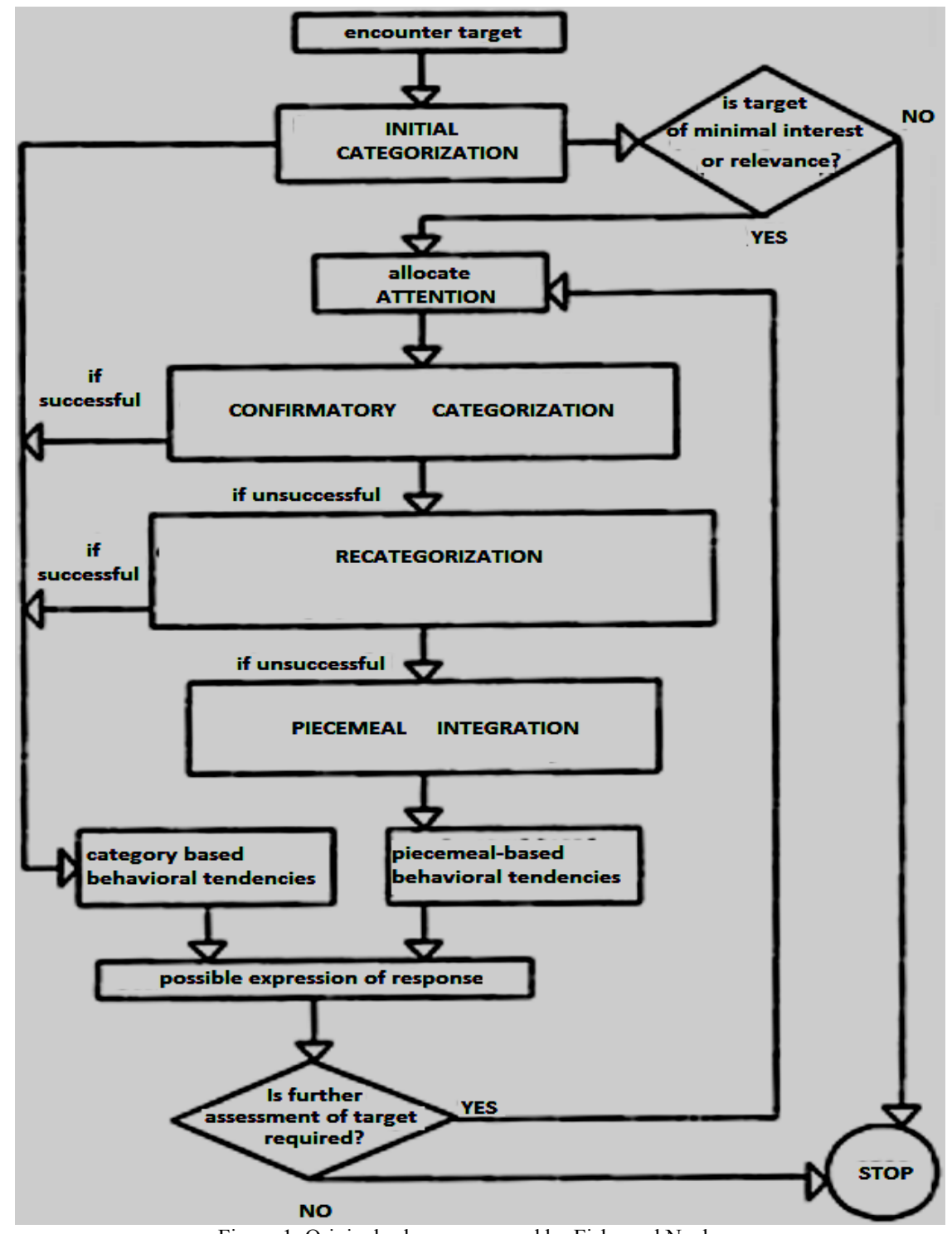

Figure 1: Original scheme proposed by Fiske and Neuberg

\section{IMPLEMENTATION}

\section{A. Recovering domestic habits from consumption data}

So far, data acquisition related to domestic activities has been carried out in two ways: asking inhabitants to write each performed action in a notebook (washing clothes, washing dishes, using an oven, etc.) or attaching an electric data logger to electrical devices. The latter solution is preferable as it offers several benefits: it does not require the inhabitants' attention, which may lead to errors in recording or approximation issues [4] and allows very precise monitoring of the usage. For instance, it is possible to distinguish between a washing machine running with a full load and a light load.

Electrical data give information about the usage of household appliances. However, it is difficult to make the distinction among habits and more complex behaviours.

Habits are typically reactive behaviours and can be defined by a reaction to a stimulus. In general, as human activities usually follow a fixed schedule each day of the week, habits can be detected through an analysis of the electric 
consumption in the time and frequency domains. In the work from Neal et al. [9], it is proposed to use the probability of receiving a reward and the frequency of an action to define its tendency to become a habit. In order to implement a computational model of Neal's definition, consumption data have to be converted to a time frequency domain instead of the time-amplitude domain used by the data logging device.

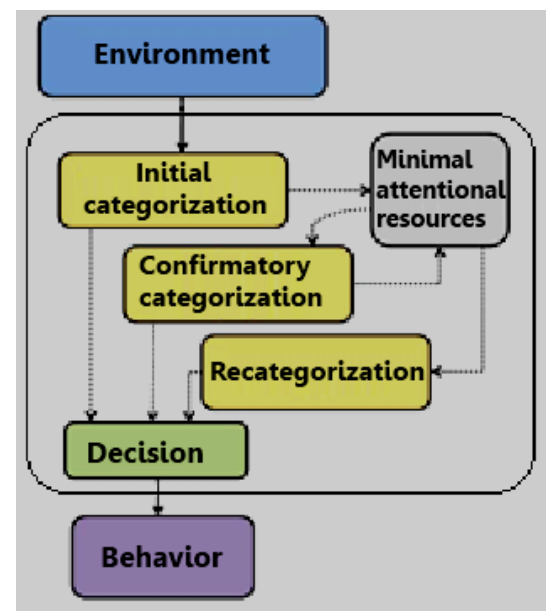

Figure 2: Proposed agent that integrates three levels of decisions

There are two ways to achieve this conversion:

The first is based on approximations of the frequencies and the second is based on the period between consecutive usage $[10,11]$. Both methods give estimates of the stability and regularity of habits, following principles of behavioural psychology. In a survey we conducted as part of the preexperimental procedures, we observed that people spontaneously describe their habits in terms of the time of performed actions and the modal period between usages. From a perception point of view, time is directly perceived by humans, not frequencies [12]. It is therefore more precise and appropriate to use the time period between events. By using periodograms, habits are easily identifiable as regions with a higher density of points (Figure 4). A continuous density estimation method is needed to determine the time period in every region of the periodogram. In order to associate higher probability regions to a higher density of points, it is suggested to use a voting method that is closely related to the kernel density estimation. Each dot representing the use of a particular device is convolved with a 2D Gaussian curve [13].

In order to detect stable habits and use them for prediction purposes, we represent usages of some domestic appliances as a continuous probability distribution function, based on time and the time period between usages for each appliance. The graphical result is a heat map representation of the periodogram. For instance, dishwasher use is shown in Figure 5. A stable habit corresponds to the region of higher probability density; in this case it can be seen that the dishwasher is mostly used once a day during the night.

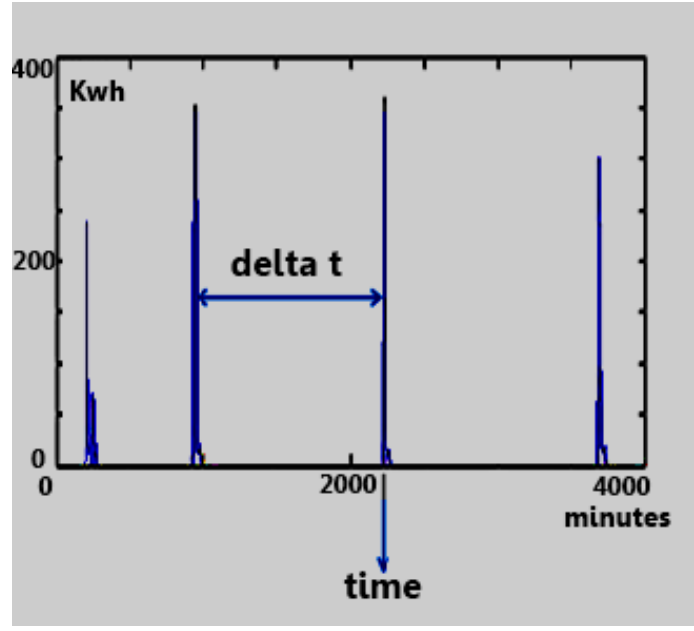

Figure 3: Raw data from the electric data logger

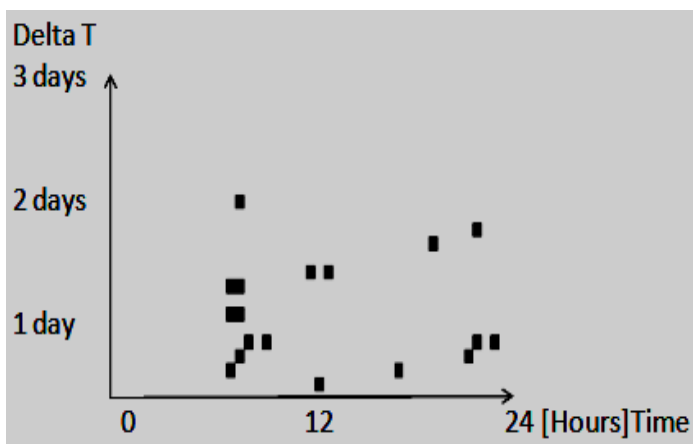

Figure 4: Events-based data in a time-period space. The abscissa delta $\mathrm{T}$ is the time in hours between utilizations, the ordinate represents the time of the utilization

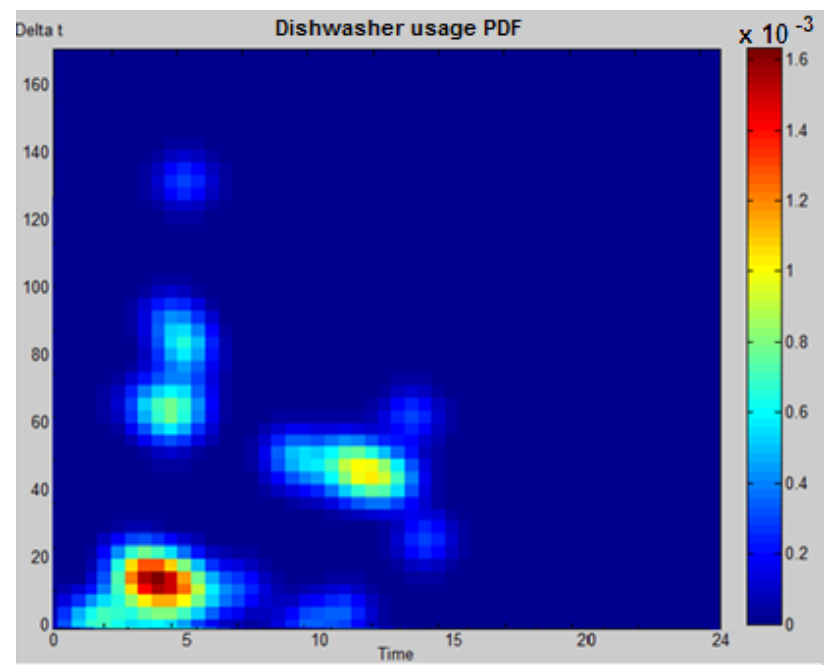

Figure 5: Probability density heat map that represents the usage of the dishwasher. The abscissa represents the time of use and the ordinate delta $\mathrm{T}$ is the duration in hours between 2 consecutive uses. 
The hotspot precisely corresponds to habits. However, areas outside the hotspot still contain useful information about domestic activities that are not habits. A possible interpretation of these areas is proposed in the next section.

\section{B. Interactions and planning of domestic activities}

A person generally decides an action after a fast evaluation of the context and the current needs. Consequently, a behaviour is usually reactive and based on habits and stereotypes (e.g., when a place is dark, the reaction is to turn on the lights). However, sometimes specific situations require a more complex categorization that takes other attributes into account that depend on the moment, past activities and the exact situation (e.g., if it is Sunday afternoon, the floor is dirty and has not been cleaned for one week, then it is time to use the vacuum cleaner). As a third possibility, humans also take adequate actions by combining the results of the previous categorizations with other considerations based on more complex reasoning processes, e.g., history of related actions, social motivations, communication with other people. It is not possible to consider all information and all complex cognitive processes involved in the decision process. We propose to focus on the variables and cognitive mechanisms that are linked to the interactions and planning of use of electric appliances.

In order to define a decision process that takes several domestic activities for planning and prioritizing into account, we need to integrate specific features such as the starting time of the activity and more global characteristics such as the correlations among activities and the variability of the time period that separates similar activities. We propose a decision process based on heat maps and several steps of categorization.

\section{Local and global features related to decisions}

One interesting way of integrating different clues into a decision process is to consider them as constraints at different time scales. With regard to planned activities, the relationship among them (i.e. the iron is turned on several hours after the washing machine) is considered as a global constraint because there is no way of precisely knowing when each action is performed. On the other hand, habits described for each action in terms of time and modal period between events are considered local constraints, as they are events localized in time and the time-period space (i.e., heat maps).

Whereas habits are located in the hotspots of heat maps, planned activities are sparser, and assumed to be found in the correlations with the local attractors.

We assume that heat maps representing usages also embed information about interactions among activities; such interactions can be considered as forms of planning. Figure 7 shows an example of this: the full representation of a usage A1 (a) includes information about habits, (b) complementary information that may be interpreted by interactions with other activities as well as some noise (c).

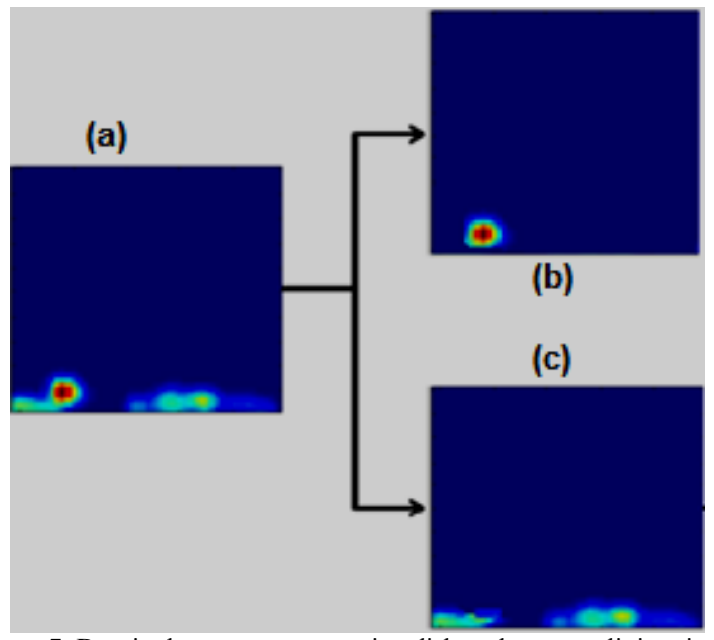

Figure 7: Density heat map representing dishwasher use split into its habits (a) and non-habits (b) components.

Interaction or planning among activities is seen as a phenomenon localized in time that links two different usages. To adequately detect this interaction and determine, for instance, if an activity $A_{2}$ is linked to $A_{1}$, heat maps of both activities have to be considered. If significant correlations are found in terms of time of use and period of use between habits of $A_{2}$ and non-habits of $A_{1}$, it is assumed that there a planning link between both activities. As illustrated in Figure $8, \mathrm{~A}_{2}$ acts as an attractor by influencing decisions concerning $A_{1}$ and helps explain some of the non-habitual actions (e). When decisions based on habits (b) and 'planned' decisions (e) are taken together, they can be used to explain most of the variance observed in the heat maps. Until now, studies usually have focused only on habits, or only on planned activities. Our framework is able to integrate both processes using the agent structure presented earlier. The algorithm of our activity simulator is presented (see algorithm 1). It iteratively generates decisions, and finally selects the likeliest action.

\section{Start}

Initialize a configuration of the consumption profiles by calculating the heat map $\mathrm{H}_{\mathrm{i}}$ for each action

\section{For each $\mathrm{H}_{i}$ do}

Generate habit $\left(\mathrm{Ha}_{\mathrm{i}}\right)$ and non-habit $\left(\mathrm{Hna}_{\mathrm{i}}\right)$ heat maps by a simple thresholding of $\mathrm{H}_{\mathrm{i}}$

$\left(\mathrm{Ha}_{\mathrm{i}}\right.$ is presented in (b) and $\mathrm{Hna}_{\mathrm{i}} \mathrm{in}(\mathrm{c})$ in igure 8)

end

For each $\mathrm{Ha}_{\mathrm{i}}, \mathrm{Hna}_{\mathrm{j}}, \mathrm{i} \neq \mathrm{jdo}$

Estimate a new weighted map $\mathbf{H i}_{\mathbf{i}}=\mathbf{H a} \mathbf{H}_{\mathbf{i}} \mathrm{Hna}_{\mathbf{j}}$, (corresponds to (e) in Figure 8)

end

end

Algorithm 1: Heat map-based habits and generation of planned activities 


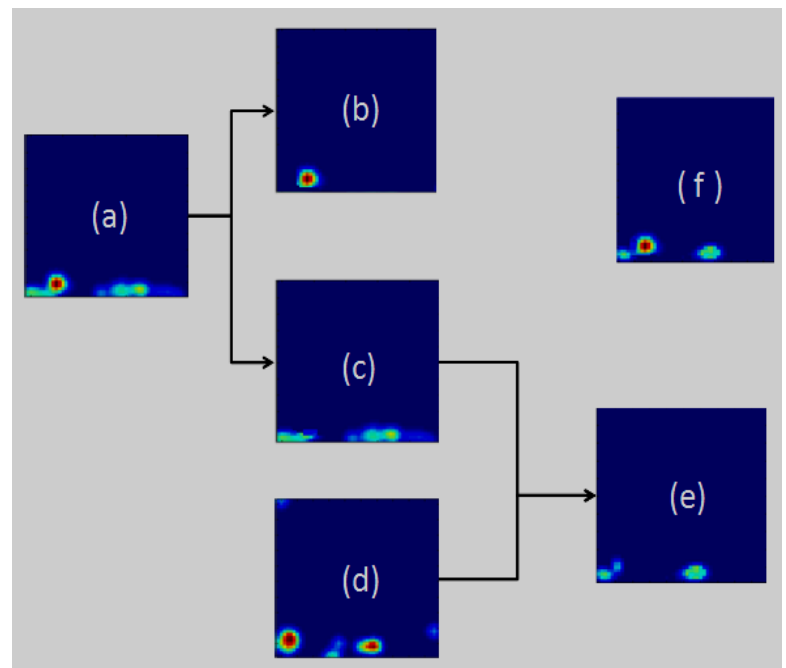

Figure 8: Heat maps representing usages contain information about planning. (a): The full representation of a usage $A_{1}$, (b): information about habits, information about non-habits (c). The full representation of activity $\mathrm{A}_{2}$ (d) is correlated with $\mathrm{A} 1$ and helps explain some non-habitual, planned actions (e). Finally, (f) shows the heat map of habits and planned instances of $A_{1}$.

\section{Calculations}

The Hadamard product, shown in equation (1), has been successfully used to take into account weighted probabilities in various contexts related to perception and discrimination [14]. Once attraction maps are calculated (heat maps linked to habits (b)), the Hadamard product is used to weigh the hotspots of each heat map with the attraction maps to calculate joint probability maps (non-habit heat maps (e)).

$$
(A \circ B)_{h, t}=A_{\ell, t} \cdot B_{\ell t}
$$

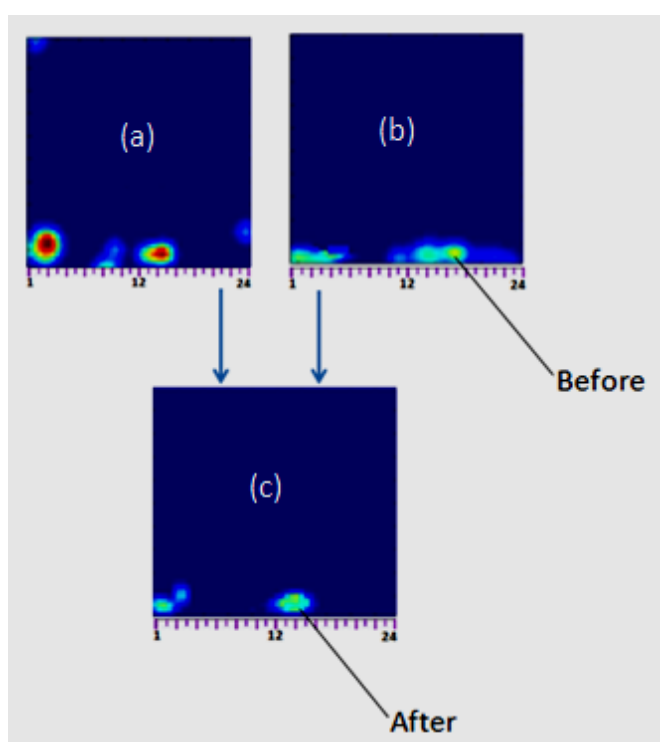

Figure 9: Heat map weighting with the Hadamard product: before the weighting procedure, most of the planned activity seems to occur around 17:00. After correction, most of planned activity is located around 14:00.
Our approach introduces local constraints that let us keep the accurately located habits in place, as opposed to a global and stronger constraint, forcing the non-habitual activity to stay within a manifold learned from the database. Figure 8 shows an example concerning a habitation where television use is linked to dishwasher use. In this case, the predictions of non-habitual dishwasher use are different when global information about television use is taken into account. Figure 9 shows the full working pipeline. When considering links between activities, predicted non-habitual usages are closer to observed usages, hence the local-global integration of information improves the quality of the simulation.

\section{E. Implementation of the decision process}

Once heat maps corresponding to habits and planned activities have been calculated, they have to be used within the agent defined in Figure 2. To do so, a probabilistic analysis has to be conducted. As a first step, domestic activities will be classified into two groups: one group with activities related to biological or physical needs (oven, dishwasher and washing machine use), the other group is related to entertainment activities (television and computer use). A mean hourly profile of usage is calculated for each group. These hourly mean profiles are used to calculate the probability of performing an activity related to entertainment or physical needs.

If the agent's first layer, annotated as 'Initial categorization' in Figure 2, detects that an action is needed, the second layer -confirmatory categorization- starts a more specific analysis.

The goal of the confirmatory categorization is to evaluate the need to act in relation to a specific task. A need is closely related to the probability of performing an action that tends to fulfil it. The probability of performing an action is given by its heat map. Once the probability of realizing each action in the database is known, a random variable (uniform distribution in $(0,1))$ is used to determine if the action is decided. For instance, if the heat map states that the probability of using the oven is 0.78 , and if $r<0.78$ then the oven is turned on. This confirmatory categorization can be seen as a behavioural variant of the Markov Chain models. The probabilities are directly linked to parameters such as the frequency and time of use, which in turn makes it possible to simulate scenarios and habits that are not registered in the database.

Confirmatory categorization is not the last step because sometimes -as suggested by the psychological model- further analysis is needed. For example, in some cases it is not possible to simultaneously turn the dishwasher and washing machine on because of the significant drop in water pressure. And conversely, as observed in our database, some people will more likely use the computer while waiting for the dishwasher to end its program. Taking these interactions among activities into account is a form of planning that is carried out by the recategorization layer.

The recategorization layer uses heat map estimations, as explained in Figure 2, in order to simulate the interaction between habits concerning action $A_{1}$ and non-habitual 
realizations of $\mathrm{A}_{2}$. Useful information concerning intentions and actions is extracted by using model-weighted maps generated by algorithm 1 . Weighted probability maps are used in a similar way. The recategorization process defines the planning part of the activities. This point is particularly important because it shows that the probabilistic features are embedded in a psychological framework. The continuum model states that a single flow of information processing may have qualitatively different results.

\section{RESULTS AND CONCLUSION}

The ENERTECH database was used to test the method. Consumption data were sampled every 10 minutes for several appliances: dishwasher, washing-machine, oven, computer and television. The database is composed of 30 different habitations with consumption data that have been gathered over a one-year period. For testing purposes, 10 different sets of data were used with a different set for learning.

Different evaluation metrics have been used in the literature to assess the quality of the simulated results [2]. The mean curves for the predicted consumptions are usually compared to the mean curves for the measured data. However this method may provide uncertainties on the quality of the predictions. Important high-frequency components that represent transient peaks of consumption may be lost. Standard metrics for signal comparison, such as PSNR (equation 3), cannot be used directly because they do not take the continuity of the days into account. For example, using PSNR, a consumption curve that has a maximum at 23:59 is considered to be very dissimilar to a curve that has a maximum at 00:01. Other techniques that allow a finer analysis, such as wavelets [8], are very accurate but computationally expensive. We propose a modified PSNR method (WPSNR for weighted-PSNR) that takes similarities in habits into account by using a weighting matrix (Figure 10). See equations (2), (3), (4), (5).

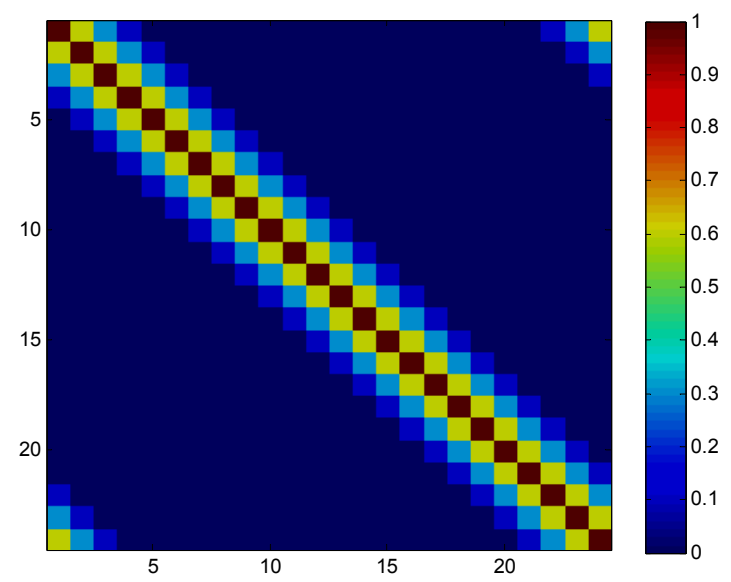

Figure 10: Weighting Matrix. $\operatorname{Dim}(w)=(24,24)$, this matrix can be used to adequately assess the similarity between generated and measured data, such as Figure 4 (a) and (f)
Let $\mathrm{S}$ be the stochastic matrix with $\mathrm{n}$ rows and $\mathrm{m}$ columns that contains all the values of the calculated heat map; $M$ is the reference (measured data).

$$
\begin{aligned}
& \text { Mean Squared Error: } \\
& \operatorname{MSE}=\frac{1}{\mathrm{~m} \mathrm{n}} \sum_{\mathrm{i}=0}^{\mathrm{m}} \sum_{\mathrm{j}=0}^{\mathrm{n}}\left((\mathrm{S}(\mathrm{i}, \mathrm{j}) \mathrm{W}-\mathrm{M}(\mathrm{i}, \mathrm{j}))^{2}\right. \\
& \text { PSNR }=10 \cdot \log \left(\frac{\text { Max range }}{M S E}\right) \\
& \text { Mean Weighted Square Error: } \\
& \text { MWSE }=\frac{1}{\mathrm{~m} \mathrm{n}} \sum_{\mathrm{i}=0}^{\mathrm{m}} \sum_{\mathrm{j}=0}^{\mathrm{n}}\left((S-M) * W *(S-M)^{T}\right) \\
& \text { Weighted PSNR: } \\
& \text { WPSNR }=10 \cdot \log \left(\frac{\text { Max range }}{\text { MWSE }}\right)
\end{aligned}
$$

WPSNR provides more accurate results than PSNR and can be quickly calculated due to the sparsity of the weighting matrix. A summary of the results is presented in Table I; as we used a weighted PSNR, the result is expressed in weighted decibels $(\mathrm{WdB})$ rather than in decibels. The predictions are generally very close to the observations. An illustration of the difference between the data and the simulation is provided in Figure 11.

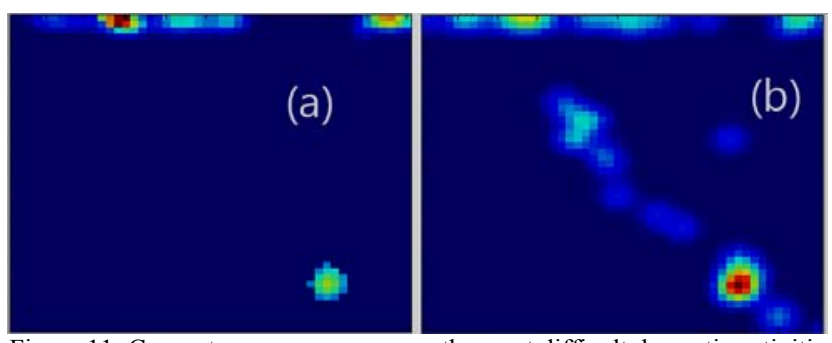

Figure 11: Computer usages are among the most difficult domestic activities to simulate. The simulation (a) is very similar to the data (b): WPSNR is 38 WdB

TABLE I. PERFormance EVALUATION

\begin{tabular}{|c|c|c|c|c|}
\hline \multirow{2}{*}{} & \multicolumn{4}{|c|}{ Table Column Head } \\
\cline { 2 - 5 } & Dishwasher & $\begin{array}{c}\text { Washing- } \\
\text { machine }\end{array}$ & Television & Computer \\
\hline WPSNR & $40 \mathrm{WdB}$ & $37 \mathrm{WdB}$ & $35 \mathrm{WdB}$ & $34 \mathrm{WdB}$ \\
\hline
\end{tabular}

As stated in the introduction, any assessment based on historical data would be biased because of the constant evolution of the different variables. The quality of the results can nevertheless be observed in Figure 4, in pictures (a) and (f). It is important to note that the proposed method is based on a psychological model of the decision process. It should therefore be more robust to the variability of the statistical data. For instance, a modification of the price of electricity diminishes the reward associated with the use of more consuming devices such as the washing machine. This 
modification can be simulated by lowering the frequency of washing habits, which corresponds to a translation of the hotspot to higher values of Delta t. Following the same method, a change in the night rate may also be simulated.

\section{REFERENCES}

[1] Balaras, C. A., Droutsa, K., Dascalaki, E., \&Kontoyiannidis, S. (2005). Heating energy consumption and resulting environmental impact of European apartment buildings. Energy and Buildings, 37(5), 429-442.

[2] Brown, R. E. (2008, July). Impact of smart grid on distribution system design. In Power and Energy Society General Meeting-Conversion and Delivery of Electrical Energy in the 21st Century, 2008 IEEE (pp. 14). IEEE.

[3] R. Page, J., Robinson, D., Morel, N., \&Scartezzini, J. L. (2008). A generalised stochastic model for the simulation of occupant presence. Energy and buildings, 40(2), 83-98.press.

[4] Widén, J., \&Wäckelgård, E. (2010). A high-resolution stochastic model of domestic activity patterns and electricity demand. Applied Energy, 87(6), 1880-1892.

[5] R. Lally, P., Van Jaarsveld, C. H., Potts, H. W., \& Wardle, J. (2010) How are habits formed: Modelling habit formation in the real world.European Journal of Social Psychology, 40(6), 998-1009.

[6] YWood, W., Quinn, J. M., \&Kashy, D. A. (2002). Habits in everyday life: thought, emotion, and action. Journal of personality and social psychology, 83(6), 1281.]
[7] Fiske, S. T., \& Neuberg, S. L. (1989). Category-based and individuating processes as a function of information and motivation: Evidence from our laboratory. In Stereotyping and prejudice (pp. 83103). Springer New York.

[8] Fiske, S. T., Lin, M., \&Neuberg, S. (1999). The continuum model. Dual-process theories in social psychology, 321-254.

[9] Neal, D. T., Wood, W., \& Quinn, J. M. (2006). Habits-A repeat performance. Current Directions in Psychological Science, 15(4), 198202.

[10] Amouroux, E., Huraux, T., Sempé, F., Sabouret, N., \& Haradji, Y. (2013). Simulating Human Activities to Investigate Household Energy Consumption. In_ICAART (2)_(pp. 71-80).

[11] Ferreri E., Salotti, J.M., \&Favier, P.A. (2014).Prediction of Electrical Consumptions Using a Bio-Inspired Behavioral Model, BauSIM2014 Conference Proceedings, Aachen, Germany, September 22-24.

[12] Richard B. Ivry, John E. Schlerf, Dedicated and intrinsic models of time perception, Trends in Cognitive Sciences, 12 (7), July 2008, Pages 273-280.

[13] Archambeau, C., Valle, M., Assenza, A., \& Verleysen, M. (2006, May). Assessment of probability density estimation methods: Parzen window and finite Gaussian mixtures. In Circuits and Systems, 2006. ISCAS 2006. Proceedings. 2006 IEEE International Symposium on (pp.4-pp)IEEE.

[14] Nicolle, J., Bailly, K., Rapp, V., \&Chetouani, M. (2013, September). Locating facial landmarks with binary map cross-correlations. In Image Processing (ICIP), $201320^{\text {th }}$ IEEE International Conference on (pp. 2978-2982). 